\title{
Decomposition of Fourth-Order Euler-Type Linear Time-Varying Differential System into Cascaded Two Second-Order Euler Commutative Pairs
}

\author{
Salisu Ibrahim $\mathbb{D D}^{1}$ and Abedallah Rababah $\mathbb{D D}^{2}$ \\ ${ }^{1}$ Department of Mathematic Education, Tishk International University-Erbil, Kurdistan Region, Erbil, Iraq \\ ${ }^{2}$ Department of Mathematical Science College of Science, United Arab Emirates University, Al Ain 15551, UAE \\ Correspondence should be addressed to Abedallah Rababah; rababah@uaeu.ac.ae
}

Received 5 July 2021; Revised 17 September 2021; Accepted 10 December 2021; Published 24 January 2022

Academic Editor: Jesus M. Munoz-Pacheco

Copyright ( $) 2022$ Salisu Ibrahim and Abedallah Rababah. This is an open access article distributed under the Creative Commons Attribution License, which permits unrestricted use, distribution, and reproduction in any medium, provided the original work is properly cited.

\begin{abstract}
This paper presents decomposition of the fourth-order Euler-type linear time-varying system (LTVS) as a commutative pair of two second-order Euler-type systems. All necessary and sufficient conditions for the decomposition are deployed to investigate the commutativity, sensitivity, and the effect of disturbance on the fourth-order LTVS. Some systems are commutative, and some are not commutative, while some are commutative under certain conditions. Based on this fact, the commutativity of fourth-order Euler-type LTVS is investigated by introducing the commutative requirements, theories, and conditions. The fourth-order Eulertype LTVSs are investigated into commutative pairs of twice Euler-type second-order linear time-varying systems (LTVSs). The decomposition theories and conditions are derived, proved, and solved to simplify the use of commutativity for practical and industrial uses. Some fourth-order systems are sensitive toward change in initial conditions or parameters while others are not, and the effect due to disturbance also varies within systems. Furthermore, the stability and robustness of systems have so many issues. But we consider fourth-order Euler-type LTVS to observe, investigate, and tackle these issues. Lastly, the realization of fourth-order LTVS from cascaded two second-order systems can be laboratory experimented which is an open problem for future engineers to investigate. However, the theoretical results show a good agreement with the simulation results is considered in this work. Perhaps it might have unlimited physical applications in science and engineering as well as theoretical contribution. But beyond any reasonable doubt, the novelty is guaranteed because this study is the first of its kind that introduces the decomposition of the fourth-order Euler-type linear time-varying system (LTVS) as a commutative pair of two second-order Euler-type systems. Illustrative examples are presented to support the results.
\end{abstract}

\section{Introduction}

The important feature of cascade-connected systems motivates scientists and engineers to use the stability analysis of the connected system for the modelling of many physical problems and designing engineering systems. The order of connection plays a vital role in realizing more stable systems in electronics and electrical engineering. Hence, the commutativity concept has a significant role in the engineering point of view. In 1977, Marshall in [1] investigated the commutativity for $1^{\text {st }}$ order continuous LTVSs. Later on, Koksal investigated the $2^{\text {nd }}$ order [2] and $3^{\text {rd }}$ order [3] continuous LTVSs. Recently, Ibrahim and Koksal in [4] investigated the commutativity and sensitivity of Euler and Onsager $6^{\text {th }}$ order LTVSs.

Decomposition is an essential mechanism that is used in many differential systems for developing the stability of a system and resolving physical problems. It is the process of splitting a high-order linear system into lower-order commutative pairs.

Decomposition formulas for $2^{\text {nd }}$ order continuous-time LTVS were proved in [5] in 2016 by Koksal. The theoretical results and application for the realization of the $4^{\text {th }}$ order LTVS were studied in [6] by Ibrahim and Koksal. 
Transitivity property of commutativity for second-order linear time-varying analogue systems was studied in [7].

When it comes to fractional-order dynamic system and experimental aspects using analogue electronic devices that are reprogrammable, the decomposition of Laplace expressions into cascaded connections of small blocks makes possible a more reliable circuit design as shown in [8]. In this work, it can be noted that the orders of the blocks matter because the filter response may mitigate some frequencies that may be required by the next block.

In this paper, the commutativity, decomposition, sensitivity, and the effect of disturbance on the fourth-order Euler-type LTVSs are considered. Furthermore, the cases of nonzero initial conditions (ICs) are considered.
This paper is organized as follows. The mathematical materials and methods that introduce the commutativity conditions are considered in Section 2. The commutative requirements and conditions along with their general solution are provided in Section 3. Results and discussions for $4^{\text {th }}$ order Euler LTVS are observed in Section 4 . Section 5 demonstrates and illustrates the effectiveness of the results by considering fourth-order Euler-type LTVS. Finally, the results are concluded in Section 6.

\section{Mathematical Materials and Methods}

Let $C$ be the fourth-order Euler LTVS, described by

$$
C: C_{4}(t) t^{4} y^{(4)}(t)+C_{3}(t) t^{3} y^{(3)}(t)+C_{2}(t) t^{2} y^{\prime \prime}(\mathrm{t})+C_{1}(t) t y^{\prime}(t)+C_{0}(t) y(t)=x(t)
$$

where the input and output are $x(t)$ and $y(t)$ and $C_{i}(t)$ represent the coefficients of the time-varying system, which are piecewise continuous functions on $\left[t_{0}, \infty\right)$. Let the initial conditions be $y\left(t_{0}\right), y^{\prime}\left(t_{0}\right), y^{\prime \prime}\left(t_{0}\right)$, and $y^{(3)}\left(t_{0}\right)$ at the initial time $t_{0} \in R$. Due to its order of $4, C_{4}(t) \equiv 0$. The decomposition of $C$ as the cascade connection of second-order systems $A$ and $B$ is given as

$$
\begin{gathered}
A: a_{2}(t) y_{A}^{\prime \prime}(t)+a_{1}(t) y_{A}^{\prime}(t)+a_{0}(t) y_{A}(t)=x_{A}(t), \\
B: b_{2}(t) y_{B}^{\prime \prime}(t)+b_{1}(t) y_{B}^{\prime}(t)+b_{0}(t) y_{B}(t)=x_{B}(t),
\end{gathered}
$$

$$
\begin{gathered}
y_{A}\left(t_{0}\right), y_{A}^{\prime}\left(t_{0}\right) . \\
y_{B}\left(t_{0}\right), y_{B}^{\prime}\left(t_{0}\right),
\end{gathered}
$$

where $a_{2}(t) \neq 0$ and $b_{2}(t) \neq 0$. Additionally, $a_{i}, b_{i}, x_{A}$, $x_{B} \in P\left[t_{0}, \infty\right)$.

The systems $A$ and $B$ are called commutative, while $(A, B)$ represents the commutative pair provided that the input-output relations of $A B$ and $B A$ are equivalent.

For the cascade connection $A B$ in Figure $1(\mathrm{a})$, the authors in [6] obtained a $4^{\text {th }}$ order LTVS for the connection $A B$ as

with ICs

$$
\begin{aligned}
& a_{2} b_{2} y^{(4)}+\left(a_{2} b_{1}+a_{1} b_{2}+2 a_{2} b_{2}^{\prime}\right) y^{(3)}+\left(a_{1} b_{1}+a_{0} b_{2}+a_{2} b_{0}+2 a_{2} b_{1}^{\prime}+a_{1} b_{2}^{\prime}+a_{2} b_{2}^{\prime \prime}\right) y^{\prime \prime} \\
& +\left(a_{0} b_{1}+a_{1} b_{0}+2 a_{2} b_{0}^{\prime}+a_{1} b_{1}^{\prime}+a_{2} b_{1}^{\prime \prime}\right) y^{\prime}+\left(a_{0} b_{0}+a_{1} b_{0}^{\prime}+a_{2} b_{0}^{\prime \prime}\right) y=x, \\
& y\left(t_{0}\right)=y_{B}\left(t_{0}\right) \\
& y^{\prime}\left(t_{0}\right)=y_{B}^{\prime}\left(t_{0}\right), \\
& y^{\prime \prime}\left(t_{0}\right)=y_{B}^{\prime \prime}\left(t_{0}\right)=\frac{y_{A}\left(t_{0}\right)-b_{0}\left(t_{0}\right) y_{B}\left(t_{0}\right)-b_{1}\left(t_{0}\right) y_{B}^{\prime}\left(t_{0}\right)}{b_{2}\left(t_{0}\right)} \\
& y^{(3)}\left(t_{0}\right)= \\
& \quad y_{B}^{(3)}\left(t_{0}\right)=\left(-\frac{b_{1}\left(t_{0}\right)}{b_{2}^{2}\left(t_{0}\right)}-\frac{b_{2}^{\prime}\left(t_{0}\right)}{b_{2}^{2}\left(t_{0}\right)}\right) y_{A}\left(t_{0}\right)+\frac{1}{b_{2}\left(t_{0}\right)} y_{A}^{\prime}\left(t_{0}\right) \\
& \quad\left(\frac{b_{0}\left(t_{0}\right) b_{1}\left(t_{0}\right)}{b_{2}^{2}\left(t_{0}\right)}+\frac{b_{0}\left(t_{0}\right) b_{2}^{\prime}\left(t_{0}\right)}{b_{2}^{2}\left(t_{0}\right)}-\frac{b_{0}^{\prime}\left(t_{0}\right)}{b_{2}\left(t_{0}\right)}\right) y_{B}\left(t_{0}\right)+\left(\frac{b_{1}^{2}\left(t_{0}\right)}{b_{2}^{2}\left(t_{0}\right)}+\frac{b_{1}\left(t_{0}\right) b_{2}^{\prime}\left(t_{0}\right)}{b_{2}^{2}\left(t_{0}\right)}-\frac{b_{0}\left(t_{0}\right)}{b_{2}\left(t_{0}\right)}-\frac{b_{1}^{\prime}\left(t_{0}\right)}{b_{2}\left(t_{0}\right)}\right) y_{B}^{\prime}\left(t_{0}\right) .
\end{aligned}
$$

Interchanging $A \leftrightarrow B$ and $a \leftrightarrow b$ from Figure 1(b), the connection BA provides the following results: 


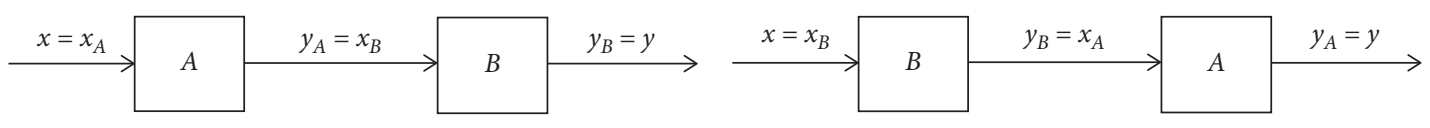

(a)

(b)

Figure 1: Cascade connection of the LTVSs.

$$
\begin{aligned}
& b_{2} a_{2} y^{(4)}+\left(b_{2} a_{1}+b_{1} a_{2}+2 b_{2} a_{2}^{\prime}\right) y^{(3)}+\left(b_{1} a_{1}+b_{0} a_{2}+b_{2} a_{0}+2 b_{2} a_{1}^{\prime}+b_{1} a_{2}^{\prime}+b_{2} a_{2}^{\prime \prime}\right) y^{\prime \prime} \\
&+\left(b_{0} a_{1}+b_{1} a_{0}+2 b_{2} a_{0}^{\prime}+b_{1} a_{1}^{\prime} b_{2} a_{1}^{\prime \prime}\right) y^{\prime}+\left(a_{0} b_{0}+b_{1} a_{0}^{\prime}+b_{2} a_{0}^{\prime \prime}\right) y=x, \\
& y\left(t_{0}\right)=y_{A}\left(t_{0}\right), \\
& y^{\prime}\left(t_{0}\right)=y_{A}^{\prime}\left(t_{0}\right), \\
& y^{\prime \prime}\left(t_{0}\right)=y_{A}^{\prime \prime}\left(t_{0}\right)=\frac{y_{B}\left(t_{0}\right)-a_{0}\left(t_{0}\right) y_{A}\left(t_{0}\right)-a_{1}\left(t_{0}\right) y_{A}^{\prime}\left(t_{0}\right)}{a_{2}\left(t_{0}\right)} \\
& y^{(3)}\left(t_{0}\right)= y_{A}^{(3)}\left(t_{0}\right)=+\left[-\frac{a_{1}\left(t_{0}\right)}{a_{2}^{2}\left(t_{0}\right)}-\frac{a_{2}^{\prime}\left(t_{0}\right)}{a_{2}^{2}\left(t_{0}\right)}\right] y_{B}\left(t_{0}\right)+\frac{1}{a_{2}\left(t_{0}\right)} y_{B}^{\prime}\left(t_{0}\right) \\
&+\left[\frac{a_{1}^{2}\left(t_{0}\right)}{a_{2}^{2}\left(t_{0}\right)}+\frac{a_{1}\left(t_{0}\right) a_{2}^{\prime}\left(t_{0}\right)}{a_{2}^{2}\left(t_{0}\right)}-\frac{a_{0}\left(t_{0}\right)}{a_{2}\left(t_{0}\right)}-\frac{a_{1}^{\prime}\left(t_{0}\right)}{a_{2}\left(t_{0}\right)}\right] y_{A}^{\prime}\left(t_{0}\right) \\
&+\left[\frac{a_{0}\left(t_{0}\right) a_{1}\left(t_{0}\right)}{a_{2}^{2}\left(t_{0}\right)}+\frac{a_{0}\left(t_{0}\right) a_{2}^{\prime}\left(t_{0}\right)}{a_{2}^{2}\left(t_{0}\right)}-\frac{a_{0}^{\prime}\left(t_{0}\right)}{a_{2}\left(t_{0}\right)}\right] y_{A}\left(t_{0}\right) . \\
&
\end{aligned}
$$

\section{Commutativity Requirements}

Two $2^{\text {nd }}$ order LTVS subsystems $A$ and $B$ are called commutative if the connections $A B$ and $B A$ are identical. Regarding this case, the equivalence is realized iff differential equations in equations (4) and (9) are the same; in addition to the initial conditions in equations (5)-(8) and equations (10)-(13) must be the same. Solving equations (4) and (9) for $b_{2}, b_{1}, b_{0}$ yields the matrix system

$$
\begin{gathered}
{\left[\begin{array}{l}
b_{2} \\
b_{1} \\
b_{0}
\end{array}\right]=\left[\begin{array}{ccc}
a_{2} & 0 & 0 \\
a_{1} & a_{2}^{0.5} & 0 \\
a_{0} & f_{32} & 1
\end{array}\right]\left[\begin{array}{l}
k_{2} \\
k_{1} \\
k_{0}
\end{array}\right],} \\
f_{32}=\frac{1}{4}\left[a_{2}^{-0.5}\left(2 a_{1}-a_{2}^{\prime}\right)\right],
\end{gathered}
$$

where $k_{2}, k_{1}, k_{0}$ are some constants. Furthermore, coefficients of the differential form of $A$ satisfy

$$
-a_{2}^{0.5} \frac{\mathrm{d}}{\mathrm{d} t}\left[a_{0}-\frac{4 a_{1}^{2}+3 \dot{a}_{2}^{2}-8 a_{1} \dot{a}_{2}+8 \dot{a}_{1} a_{2}-4 a_{2} \ddot{a}_{2}}{16 a_{2}}\right] k_{1}=0, \forall t \geq t_{0} .
$$

Commutativity with arbitrary and nonzero initial conditions can be assured by considering equations (16)-(22) (for more details, see [6]):

$$
\begin{aligned}
& y=y_{B}=y_{A}, \\
& y^{\prime}=y_{B}^{\prime}=y_{A}^{\prime}, \\
& y^{\prime \prime}=\left(\frac{1}{b_{2}}-\frac{b_{0}}{b_{2}}\right) y_{A}-\frac{b_{1} y_{A}^{\prime}}{b_{2}}=\left(\frac{1}{a_{2}}-\frac{a_{0}}{a_{2}}\right) y_{A}-\frac{a_{1} y_{A}^{\prime}}{a_{2}},
\end{aligned}
$$




$$
\begin{aligned}
& y^{(3)}=\left(-\frac{b_{1}}{b_{2}^{2}}-\frac{b_{2}^{\prime}}{b_{2}^{2}}\right) y_{A}+\left(\frac{b_{0} b_{1}}{b_{2}^{2}}-\frac{b_{0}^{\prime}}{b_{2}}+\frac{b_{0} b_{2}^{\prime}}{b_{2}^{2}}\right) y_{B}+\frac{1}{b_{2}} y_{A}^{\prime}+\left(\frac{b_{1}^{2}}{b_{2}^{2}}-\frac{b_{0}}{b_{2}}-\frac{b_{1}^{\prime}}{b_{2}}+\frac{b_{1} b_{2}^{\prime}}{b_{2}^{2}}\right), \\
& =\left(\frac{a_{0} a_{1}}{a_{2}^{2}}-\frac{a_{0}^{\prime}}{a_{2}}+\frac{a_{0} a_{2}^{\prime}}{a_{2}^{2}}\right) y_{A}+\left(\frac{a_{1}^{2}}{a_{2}^{2}}-\frac{a_{0}}{a_{2}}-\frac{a_{1}^{\prime}}{a_{2}}+\frac{a_{1} a_{2}^{\prime}}{a_{2}^{2}}\right) y_{A}^{\prime}+\left(-\frac{a_{1}}{a_{2}^{2}}-\frac{a_{2}^{\prime}}{a_{2}^{2}}\right) y_{B}+\frac{1}{a_{2}} y_{B}^{\prime} \text {, } \\
& \left(-\frac{1}{a_{2}}+\frac{1}{a_{2} k_{2}}-\frac{k_{0}}{a_{2} k_{2}}-\frac{a_{1} k_{1}}{2 a_{2}^{3 / 2} k_{2}}+\frac{k_{1} a_{2}^{\prime}}{4 a_{2}^{3 / 2} k_{2}}\right) y_{A}-\frac{k_{1} y_{A}^{\prime}}{\sqrt{a_{2}} k_{2}}=0, \\
& {\left[\begin{array}{c}
\frac{a_{1}}{a_{2}^{2}}-\frac{k_{1}}{a_{2}^{3 / 2} k_{2}^{2}}-\frac{a_{1}}{a_{2}^{2} k_{2}}+\frac{k_{0} k_{1}}{a_{2}^{3 / 2} k_{2}^{2}}+\frac{a_{1} k_{1}^{2}}{2 a_{2}^{2} k_{2}^{2}}+\frac{a_{1} k_{0}}{a_{2}^{2} k_{2}}+\frac{a_{1}^{2} k_{1}}{2 a_{2}^{5 / 2} k_{2}}+\frac{a_{0} k_{1}}{a_{2}^{3 / 2} k_{2}} \\
\frac{k_{1} a_{1}^{\prime}}{2 a_{2}^{3 / 2} k_{2}}+\frac{a_{2}^{\prime}}{a_{2}^{2}}-\frac{a_{2}^{\prime}}{a_{2}^{2} k_{2}}+\frac{a_{1} k_{1} a_{2}^{\prime}}{2 a_{2}^{5 / 2} k_{2}}-\frac{k_{1}^{2} a_{2}^{\prime}}{4 a_{2}^{2} k_{2}^{2}}+\frac{k_{0} a_{2}^{\prime}}{a_{2}^{2} k_{2}}-\frac{3 k_{1}\left(a_{2}^{\prime}\right)^{2}}{8 a_{2}^{5 / 2} k_{2}}+\frac{k_{1} a_{1}^{\prime \prime}}{4 a_{2}^{3 / 2} k_{2}}
\end{array}\right] y_{A}} \\
& +\left(-\frac{1}{a_{2}}+\frac{k_{1}^{2}}{a_{2} k_{2}^{2}}+\frac{1}{a_{2} k_{2}}+\frac{3 a_{1} k_{1}}{2 a_{2}^{3 / 2} k_{2}}-\frac{k_{0}}{a_{2} k_{2}}+\frac{3 k_{1} a_{2}^{\prime}}{4 a_{2}^{3 / 2} k_{2}}\right) y_{A}^{\prime}=0, \\
& \left(k_{0}+k_{2}-1\right)=0 \text {. }
\end{aligned}
$$

\section{Results and Discussion}

In this section, the explicit commutative formulas obtained from the previous section are considered, and the coefficients of the decompositions $A$ and $B$ are expressed in terms of the decomposed fourth-order Euler system $C$. Observe that (1) and (4) are both $4^{\text {th }}$ order LTVSs, and the equivalent relation between them leads to

$$
a_{2} b_{2}=C_{4} t^{4}=a_{2}^{2} k_{2}=C_{4} t^{4} \longrightarrow a_{2}=t^{2}\left(\frac{C_{4}}{k_{2}}\right)^{1 / 2} \text {. }
$$

Comparing the coefficients of the $3^{\text {rd }}$ derivatives and making use of (14) leads to

$$
a_{1}=\frac{1}{2}\left[-\frac{C_{4}^{\prime}}{\sqrt{C_{4}} \sqrt{k_{2}}} t^{2}+\left(-\frac{C_{4}^{1 / 4} k_{1}}{k_{2}^{5 / 4}}+\frac{C_{3}}{\sqrt{C_{4}} \sqrt{k_{2}}}-\frac{4 \sqrt{C_{4}}}{\sqrt{k_{2}}}\right) t\right] .
$$

Relating the coefficients of the $2^{\text {nd }}$ derivatives and making use of (14) gives

$$
a_{0}=\frac{1}{16}\left[\begin{array}{c}
\left(\frac{2\left(C_{4}^{\prime}\right)^{2}}{C_{4}^{3 / 2} k_{2}^{3 / 4}}-\frac{4\left(C_{4}^{\prime}\right)^{2}}{C_{4}^{3 / 2} \sqrt{k_{2}}}-\frac{4 C_{4}^{\prime \prime}}{\sqrt{C_{4}} k_{2}^{3 / 4}}+\frac{8 C_{4}^{\prime \prime}}{\sqrt{C_{4}} \sqrt{k_{2}}}\right) t^{2} \\
+\left(-\frac{8 C_{3}^{\prime}}{\sqrt{C_{4}} \sqrt{k_{2}}}+\frac{3 k_{1} C_{4}^{\prime}}{C_{4}^{3 / 4} k_{2}^{5 / 4}}-\frac{16 C_{4}^{\prime}}{\sqrt{C_{4}} k_{2}^{3 / 4}}+\frac{6 C_{3} C_{4}^{\prime}}{C_{4}^{3 / 2} \sqrt{k_{2}}}+\frac{32 C_{4}^{\prime}}{\sqrt{C_{4}} \sqrt{k_{2}}}\right) t \\
+\frac{4 k_{1}^{2}}{k_{2}^{2}}-\frac{2 C_{3} k_{1}}{C_{4}^{3 / 4} k_{2}^{5 / 4}}+\frac{12 C_{4}^{1 / 4} k_{1}}{k_{2}^{5 / 4}}-\frac{16 \sqrt{C_{4}}}{k_{2}^{3 / 4}}-\frac{2 C_{3}^{2}}{C_{4}^{3 / 2} \sqrt{k_{2}}}+\frac{8 C_{2}}{\sqrt{C_{4}} \sqrt{k_{2}}}+\frac{32 \sqrt{C_{4}}}{\sqrt{k_{2}}}-\frac{8 k_{0}}{k_{2}}
\end{array}\right] .
$$

Considering the obtained results $a_{2}, a_{1}$, and $a_{0}$ of equations (23)-(25), respectively, we figure out $b_{2}, b_{1}, b_{0}$ using (14) and present them in the following explicit forms: 


$$
\begin{aligned}
& b_{2}=k_{2}^{1 / 2} c_{4}^{1 / 2} t^{2}, \\
& b_{1}=\frac{1}{2}\left[-\frac{\sqrt{k_{2}} C_{4}^{\prime}}{\sqrt{C_{4}}} t^{2}+\left(\frac{C_{4}^{1 / 4} k_{1}}{k_{2}^{1 / 4}}+\frac{C_{3} \sqrt{k_{2}}}{\sqrt{C_{4}}}-4 \sqrt{C_{4}} \sqrt{k_{2}}\right) t\right] \text {, } \\
& b_{0}=\frac{1}{16}\left[\begin{array}{c}
\left(\frac{2 k_{2}^{1 / 4}\left(C_{4}^{\prime}\right)^{2}}{C_{4}^{3 / 2}}-\frac{4 \sqrt{k_{2}}\left(C_{4}^{\prime}\right)^{2}}{C_{4}^{3 / 2}}-\frac{4 k_{2}^{1 / 4} C_{4}^{\prime \prime}}{\sqrt{C_{4}}}+\frac{8 \sqrt{k_{2}} C_{4}^{\prime \prime}}{\sqrt{C_{4}}}\right) t^{2} \\
+\left(\frac{8 \sqrt{k_{2}} C_{3}^{\prime}}{\sqrt{C_{4}}}-\frac{3 k_{1} C_{4}^{\prime}}{C_{4}^{3 / 4} k_{2}^{1 / 4}}-\frac{16 k_{2}^{1 / 4} C_{4}^{\prime}}{\sqrt{C_{4}}}+\frac{6 C_{3} \sqrt{k_{2}} C_{4}^{\prime}}{C_{4}^{3 / 2}}+\frac{32 \sqrt{k_{2}} C_{4}^{\prime}}{\sqrt{C_{4}}}\right) t+8 k_{0} \\
+\frac{2 C_{3} k_{1}}{C_{4}^{3 / 4} k_{2}^{1 / 4}}-\frac{12 C_{4}^{1 / 4} k_{1}}{k_{2}^{1 / 4}}-16 \sqrt{C_{4}} k_{2}^{1 / 4}-\frac{2 C_{3}^{2} \sqrt{k_{2}}}{C_{4}^{3 / 2}}+\frac{8 C_{2} \sqrt{k_{2}}}{\sqrt{C_{4}}}+32 \sqrt{C_{4}} \sqrt{k_{2}}
\end{array}\right] .
\end{aligned}
$$

From (1) and (4), one needs to observe the following equations:

$$
\begin{aligned}
t C_{1} & =a_{0} b_{1}+a_{1} b_{0}+2 a_{2} b_{0}^{\prime}+a_{1} b_{1}^{\prime}+a_{2} b_{1}^{\prime \prime}, \\
C_{0} & =\left(a_{0} b_{0}+a_{1} b_{0}^{\prime}+a_{2} b_{0}^{\prime \prime}\right) .
\end{aligned}
$$

Substituting the coefficients $a_{2}, a_{1}, a_{0}, b_{2}, b_{1}$, and $b_{0}$ from (23)-(28) into (29) and (30), after numerous mathematical computations and comparative analysis, the following requirements are acquired:

$$
\begin{gathered}
C_{1}=\frac{1}{8}\left(-16 C_{2}-\frac{C_{3}^{3}}{C_{4}^{2}}+\frac{4 C_{2} C_{3}}{C_{4}}+\frac{6 C_{3}^{2}}{C_{4}}-32 C_{4}+\frac{C_{4}^{1 / 4} k_{1}^{3}}{k_{2}^{9 / 4}}-\frac{8 C_{3}}{k_{2}^{1 / 4}}+\frac{32 C_{4}}{k_{2}^{1 / 4}}-\frac{4 C_{4}^{1 / 4} k_{0} k_{1}}{k_{2}^{5 / 4}}\right), \\
C_{0}=\frac{1}{256}\left(\begin{array}{c}
5512 C_{2}+\frac{4 C_{3}^{4}}{C_{4}^{3}}-\frac{32 C_{2} C_{3}^{2}}{C_{4}^{2}}+\frac{64 C_{2}^{2}}{C_{4}}-\frac{128 C_{3}^{2}}{C_{4}}+1024 C_{4}+\frac{8 C_{3} k_{1}^{3}}{C_{4}^{3 / 2} k_{2}^{9 / 4}}-\frac{48 C_{4}^{1 / 4} k_{1}^{3}}{k_{2}^{9 / 4}} \\
+\frac{32 k_{0} k_{1}^{2}}{k_{2}^{2}}-\frac{64 \sqrt{C_{4}} k_{1}^{2}}{k_{2}^{7 / 4}}-\frac{12 C_{3}^{2} k_{1}^{2}}{C_{4}^{3 / 2} k_{2}^{3 / 2}}+\frac{32 C_{2} k_{1}^{2}}{\sqrt{C_{4}} k_{2}^{3 / 2}}+\frac{48 C_{3} k_{1}^{2}}{\sqrt{C_{4}} k_{2}^{3 / 2}}-\frac{16 \sqrt{C_{4}} k_{1}^{2}}{k_{2}^{3 / 2}}-\frac{32 C_{3} k_{0} k_{1}}{C_{4}^{3 / 4} k_{2}^{5 / 4}} \\
+\frac{192 C_{4}^{1 / 4} k_{0} k_{1}}{k_{2}^{5 / 4}}-\frac{64 k_{0}^{2}}{k_{2}}+\frac{256 C_{4}}{\sqrt{k_{2}}}-\frac{256 C_{2}}{k_{2}^{1 / 4}}+\frac{64 C_{3}^{2}}{C_{4} k_{2}^{1 / 4}}-\frac{1024 C_{4}}{k_{2}^{1 / 4}}
\end{array}\right) .
\end{gathered}
$$

Theorem 1. The necessary and sufficient conditions for Euler 4th-order LTVS of equation (1) to be decomposed into cascade-connected LTV commutative pairs of Euler 2nd-order are that

(i) There exist some constants $k_{2}, k_{1}, k_{0}$ such that the coefficients $C_{1}$ and $C_{0}$ can be formulated in connection with $C_{4}, C_{3}$, and $C_{2}$ as in (31) and (32), respectively,

(ii) The reduced Euler $2^{\text {nd }}$ order $A$ and $B$ must be formulated with regard to $C_{4}, C_{3}$, and $C_{2}$ as in equations (23)-(28).

Proof. The detailed proof of (i) of Theorem 1 can be seen in equations (29) and (30), while in (ii) of Theorem 1, equations
(23)-(28) are obtained from the coefficients of equations (1) and (4).

Note. Theorem 1 is applicable to the zero IC case. Regarding the case of nonzero ICs, it is covered in Theorem 2.

Theorem 2. The necessary and sufficient conditions for the decomposition of Euler $4^{\text {th }}$ order LTVS C with nonzero ICs into its twin Euler $2^{\text {nd }}$ order LTV commutative pairs $A$ and $B$ are that

(i) The requirements of Theorem 1 are fulfilled.

(ii) The ICs of A, B, C must obey equations (16) and (17).

(iii) Furthermore, the ICs of A must obey equations (20) and (21).

(iv) In addition, the ICs of $C$ must be satisfied. 


$$
\begin{aligned}
& y^{\prime \prime}\left(t_{0}\right)=\left[\begin{array}{c}
-\frac{2}{t^{2}}+\frac{C_{3}^{2}}{8 t^{2} C_{4}^{2}}-\frac{C_{2}}{2 t^{2} C_{4}}-\frac{k_{1}^{2}}{4 t^{2} \sqrt{C_{4}} k_{2}^{3 / 2}}+\frac{C_{3} k_{1}}{8 t^{2} C_{4}^{5 / 4} k_{2}^{3 / 4}}-\frac{3 k_{1}}{4 t^{2} C_{4}^{1 / 4} k_{2}^{3 / 4}}+\frac{k_{0}}{2 t^{2} \sqrt{C_{4}} \sqrt{k_{2}}}+ \\
\frac{1}{t^{2} k_{2}^{1 / 4}}+\frac{\sqrt{k_{2}}}{t^{2} \sqrt{C_{4}}}+\frac{C_{3}^{\prime}}{2 t C_{4}}-\frac{3 C_{3} C_{4}^{\prime}}{8 t C_{4}^{2}}-\frac{2 C_{4}^{\prime}}{t C_{4}}-\frac{3 k_{1} C_{4}^{\prime}}{16 t C_{4}^{5 / 4} k_{2}^{3 / 4}}+\frac{C_{4}^{\prime}}{t C_{4} k_{2}^{1 / 4}}+\frac{\left(C_{4}^{\prime}\right)^{2}}{4 C_{4}^{2}}-\frac{\left(C_{4}^{\prime}\right)^{2}}{8 C_{4}^{2} k_{2}^{1 / 4}}-\frac{C_{4}^{\prime \prime}}{2 C_{4}}+\frac{C_{4}^{\prime \prime}}{4 C_{4} k_{2}^{1 / 4}}
\end{array}\right] y_{A}\left(t_{0}\right), \\
& {\left[-\frac{C_{3}^{3}}{16 t^{3} C_{4}^{3}}+\frac{C_{2} C_{3}}{4 t^{3} C_{4}^{2}}+\frac{C_{3}}{t^{3} C_{4}}-\frac{k_{1}^{3}}{8 t^{3} C_{4}^{3 / 4} k_{2}^{9 / 4}}+\frac{3 C_{3} k_{1}^{2}}{16 t^{3} C_{4}^{3 / 2} k_{2}^{3 / 2}}-\frac{3 k_{1}^{2}}{8 t^{3} \sqrt{C_{4}} k_{2}^{3 / 2}}+\frac{k_{0} k_{1}}{4 t^{3} C_{4}^{3 / 4} k_{2}^{5 / 4}}\right.} \\
& +\frac{k_{1}}{2 t^{3} C_{4}^{1 / 4} k_{2}}-\frac{C_{2} k_{1}}{4 t^{3} C_{4}^{5 / 4} k_{2}^{3 / 4}}+\frac{3 C_{3} k_{1}}{8 t^{3} C_{4}^{5 / 4} k_{2}^{3 / 4}}-\frac{k_{1}}{t^{3} C_{4}^{1 / 4} k_{2}^{3 / 4}}-\frac{C_{3} k_{0}}{4 t^{3} C_{4}^{3 / 2} \sqrt{k_{2}}}-\frac{C_{3}}{2 t^{3} C_{4} k_{2}^{1 / 4}} \\
& y^{(3)}\left(t_{0}\right)=\left[\begin{array}{c}
+\frac{k_{1}}{2 t^{3} C_{4}^{3 / 4} k_{2}^{1 / 4}}-\frac{C_{3} \sqrt{k_{2}}}{2 t^{3} C_{4}^{3 / 2}}-\frac{C_{2}^{\prime}}{2 t^{2} C_{4}}-\frac{C_{3} C_{3}^{\prime}}{4 t^{2} C_{4}^{2}}+\frac{C_{3}^{\prime}}{2 t^{2} C_{4}}+\frac{3 k_{1} C_{3}^{\prime}}{8 t^{2} C_{4}^{5 / 4} k_{2}^{3 / 4}}+\frac{C_{3} \sqrt{k_{2}} C_{3}^{\prime}}{4 t^{2} C_{4}^{2}} \\
+\frac{3 C_{3}^{2} C_{4}^{\prime}}{16 t^{2} C_{4}^{3}}+\frac{C_{2} C_{4}^{\prime}}{4 t^{2} C_{4}^{2}}+\frac{5 C_{3} C_{4}^{\prime}}{8 t^{2} C_{4}^{2}}-\frac{3 C_{4}^{\prime}}{t^{2} C_{4}}-\frac{3 k_{1}^{2} C_{4}^{\prime}}{32 t^{2} C_{4}^{3 / 2} k_{2}^{3 / 2}}+\frac{k_{1} C_{4}^{\prime}}{2 t^{2} C_{4}^{5 / 4} k_{2}}-\frac{3 C_{3} k_{1} C_{4}^{\prime}}{16 t^{2} C_{4}^{9 / 4} k_{2}^{3 / 4}}+\frac{3 C_{4}^{\prime}}{2 t^{2} C_{4} k_{2}^{1 / 4}}-\frac{11 k_{1} C_{4}^{\prime}}{8 t^{2} C_{4}^{5 / 4} k_{2}^{3 / 4}}
\end{array}\right. \\
& {\left[-\frac{C_{3} C_{4}^{\prime}}{2 t^{2} C_{4}^{2} k_{2}^{1 / 4}}-\frac{3 C_{3}^{2} \sqrt{k_{2}} C_{4}^{\prime}}{16 t^{2} C_{4}^{3}}-\frac{5 C_{3}^{\prime} C_{4}^{\prime}}{8 t C_{4}^{2}}+\frac{7 C_{3}\left(C_{4}^{\prime}\right)^{2}}{16 t C_{4}^{3}}+\frac{3\left(C_{4}^{\prime}\right)^{2}}{2 t C_{4}^{2}}-\frac{k_{1}\left(C_{4}^{\prime}\right)^{2}}{16 t C_{4}^{9 / 4} k_{2}}+\frac{17 k_{1}\left(C_{4}^{\prime}\right)^{2}}{64 t C_{4}^{9 / 4} k_{2}^{3 / 4}}+\frac{C_{3}\left(C_{4}^{\prime}\right)^{2}}{16 t C_{4}^{3} k_{2}^{1 / 4}}-\frac{\left(C_{4}^{\prime}\right)^{2}}{4 t C_{4}^{2} k_{2}^{1 / 4}}-\frac{3\left(C_{4}^{\prime}\right)^{3}}{8 C_{4}^{3}}\right]} \\
& +\frac{3\left(C_{4}^{\prime}\right)^{3}}{16 C_{4}^{3} k_{2}^{1 / 4}}+\frac{C_{4}^{\prime \prime}}{2 t C_{4}}-\frac{C_{3} C_{4}^{\prime \prime}}{8 t C_{4}^{2}}-\frac{3 C_{4}^{\prime \prime}}{t C_{4}}+\frac{k_{1} C_{4}^{\prime \prime}}{8 t C_{4}^{5 / 4} k_{2}}-\frac{7 k_{1} C_{4}^{\prime \prime}}{16 t C_{4}^{5 / 4} k_{2}^{3 / 4}}-\frac{C_{3} C_{4}^{\prime \prime}}{8 t C_{4}^{2} k_{2}^{1 / 4}}+\frac{3 C_{4}^{\prime \prime}}{2 t C_{4} k_{2}^{1 / 4}}+\frac{3 C_{4}^{\prime} C_{4}^{\prime \prime}}{4 C_{4}^{2}}-\frac{3 C_{4}^{\prime} C_{4}^{\prime \prime}}{8 C_{4}^{2} k_{2}^{1 / 4}}-\frac{C_{4}^{(3)}}{2 C_{4}}+\frac{C_{4}^{(3)}}{4 C_{4} k_{2}^{1 / 4}} \\
& +\left[\begin{array}{c}
{\left[\frac{3 C_{3}^{2}}{8 t^{2} C_{4}^{2}}-\frac{C_{2}}{2 t^{2} C_{4}}-\frac{3 C_{3}}{2 t^{2} C_{4}}-\frac{3 C_{3} k_{1}}{8 t^{2} C_{4}^{5 / 4} k_{2}^{3 / 4}}+\frac{1}{t^{2} k_{2}^{1 / 4}}+\frac{3 k_{1}}{4 t^{2} C_{4}^{1 / 4} k_{2}^{3 / 4}}+\frac{k_{0}}{2 t^{2} \sqrt{C_{4}} \sqrt{k_{2}}}\right.} \\
+\frac{\sqrt{k_{2}}}{t^{2} \sqrt{C_{4}}}-\frac{3 C_{3} C_{4}^{\prime}}{8 t C_{4}^{2}}+\frac{3 k_{1} C_{4}^{\prime}}{16 t C_{4}^{5 / 4} k_{2}^{3 / 4}}+\frac{C_{4}^{\prime}}{t C_{4} k_{2}^{1 / 4}}-\frac{\left(C_{4}^{\prime}\right)^{2}}{8 C_{4}^{2} k_{2}^{1 / 4}}+\frac{C_{4}^{\prime \prime}}{4 C_{4} k_{2}^{1 / 4}}
\end{array}\right] y_{A}^{\prime}\left(t_{0}\right) .
\end{aligned}
$$

(v) For $k_{1}=0$, the ICs must satisfy (22).

Proof. Theorem 2 (i) is obvious; for Theorem 2 (ii), the ICs in equations (16) and (17) are obtained from equations (5), (10) and equation (6), (11), respectively. Patterning Theorem 2(iii), the ICs in equations (20) and (21) are obtained from equations (7), (12) and equations (8), (13), respectively.

Regarding equation (33), we consider equation (18); inserting in values of $a_{2}, a_{1}$, and $a_{0}$ of equations (23)-(25) leads to equation (33). Equation (34) is obtained by using equation (19); substituting the values of $a_{2}, a_{1}$, and $a_{0}$ of equations (23)-(25), respectively, in equation (19) provides equation (34). Item (v) results from equation (22) as the solution of equations (20) and (21).

\section{Applications to Fourth-Order Euler-Type LTVS}

We apply the results obtained from the previous sections to investigate the decomposition of fourth-order Euler LTVS as a commutative pair of two second-order Euler-type systems.
5.1. Example 1. Considering the constants $C_{4}=C_{3}=C_{2}=$ 1 , with $k_{i}^{\prime} s$ in equation (36), $C_{1}=-15 / 8$ and $C_{0}=299 / 192$ are obtained by making use of equations (31) and (32), respectively. The general Euler-type fourth-order LTVS $C$ of equation (1) becomes

$$
t^{4} y^{(4)}(t)+t^{3} y^{(3)}(t)+t^{2} y^{\prime \prime}(t)-\frac{15}{8} t y^{\prime}(t)+\frac{299}{192} y(t)=x(t) .
$$

Note that equation (22) is satisfied with the constants

$$
k_{2}=3, k_{1}=0, k_{0}=-2 .
$$

Furthermore, equations (16)-(19) lead to

$$
\begin{aligned}
& y_{B}\left(t_{0}\right)=y_{A}\left(t_{0}\right)=y\left(t_{0}\right), \\
& y_{B}^{\prime}\left(t_{0}\right)=y_{A}^{\prime}\left(t_{0}\right)=y^{\prime}\left(t_{0}\right), \\
& y^{\prime \prime}\left(t_{0}\right)=\left(-\frac{11}{8}+\frac{2}{\sqrt{3}}\right) y\left(t_{0}\right)+\frac{3}{2} y^{\prime}\left(t_{0}\right) \text { for } t_{0}=1,
\end{aligned}
$$




$$
y^{(3)}\left(t_{0}\right)=\left(\frac{11}{16}-\frac{1}{\sqrt{3}}\right) y\left(t_{0}\right)+\left(-\frac{5}{8}+\frac{2}{\sqrt{3}}\right) y^{\prime}\left(t_{0}\right) \text { for } t_{0}=1
$$

The Euler subsystems $A$ and $B$ generate the equations

$$
A: \frac{t^{2}}{\sqrt{3}} y_{A}^{\prime \prime}(t)-\frac{\sqrt{3}}{2} t y_{A}^{\prime}(t)+\left(\frac{33+8 \sqrt{3}}{24 \sqrt{3}}\right) y_{A}(t)=x_{A}(t),
$$

$$
B: \sqrt{3} t^{2} y_{B}^{\prime}(t)-\frac{3 \sqrt{3}}{2} t y_{B}^{\prime}(t)+\left(\frac{11 \sqrt{3}-8}{8}\right) y_{B}(t)=x_{B}(t) .
$$

Note that both subsystems A and B in the decomposition are of Euler type. Simulation was carried out with a sinusoid of frequency $3 \mathrm{rad} / \mathrm{sec}$, amplitude 2, and bias $-21 / 10$, considering ode (Bogacki-Shampine) as the solver, while maintaining a fixed step length of 0.01 . Simulink outcomes are illustrated in Figure 2. The initial time and initial states chosen are $t_{0}=1$ and $y(1)=y_{A}(1)=y_{B}(1)=y_{B}^{\prime}(1)=y_{A}^{\prime}$ $(1)=y^{\prime}(1)=0.5$. Equations (39) and (40) lead to $y^{\prime \prime}(1)=1 / 16+1 / \sqrt{3}, y^{(3)}(1)=1 / 32+1 / 2 \sqrt{3}$. After satisfying all decomposition conditions, the systems $A B, B A$, and $C$ produce a similar response $(B=B A=C$, see Figure 2). However, with a small modification in the decomposition requirement by changing $y_{B}(1)=y_{A}(1)=y(1)=0.5$ to $y_{A}(1)=y(1)=0.6$, then equation (37) is not satisfied, and the decomposition is messed up, and it is not valid any more (see $A B 1, B A 1, C 1$ in Figure 2). Observe that $A B 1$ is slightly disturbed by the changes, while $B A 1$ is so sensitive to ICs. Therefore, A and B should be connected in the cascade synthesis of $\mathrm{C}$.

5.2. Example 2. In this example, the $4^{\text {th }}$ order LTVS $\mathrm{C}$ is reconstructed by considering the $2^{\text {nd }}$ order subsystem A. In line with this, consider the $2^{\text {nd }}$ order LTVS $A$ defined by

$$
\text { A: } y_{A}^{\prime \prime}(t)+\sin (t) y_{A}^{\prime}(t)+\left(\frac{\sin ^{2}(t)}{4}+\frac{\sin (t)}{\sqrt{5}}+\frac{\cos (t)}{2}+\frac{3}{4}\right) y_{A}(t)=x_{A}(t)
$$

For the constant $k_{1}$ :

$$
\frac{1}{k_{2}^{2}}\left[\begin{array}{cc}
k_{2}-k_{2} k_{0}-k_{2}^{2}-0.5 k_{2} k_{1} t & -k_{1} k_{2} \\
k_{0} k_{1}-k_{1}-0.5 k_{2} k_{1}+V & k_{1}^{2}+k_{2}-k_{2} k_{0}-k_{2}^{2}+1.5 k_{2} k_{1} t
\end{array}\right]\left[\begin{array}{l}
y_{A} \\
y_{A}^{\prime}
\end{array}\right]=0
$$

where $V=\left(1-k_{2}+0.5 k_{1}^{2}+k_{2} k_{0}\right) t+0.25 k_{2} k_{1} t^{2}$.

For the commutativity with nonzero ICs, the coefficient matrix in equation (44) must be singular at $t_{0}=0$, that is, its determinant is zero if

$$
k_{1}=\mp \frac{2}{\sqrt{5}}\left(k_{0}+k_{2}-1\right) .
$$

Furthermore, equation (44) at $t_{0}=0$ requires

$$
\left(k_{2}-k_{2} k_{0}-k_{2}^{2}\right) y_{A}-k_{1} k_{2} y_{A}^{\prime}=0 .
$$

Inserting equation (45) into equation (46) guaranties commutativity of $A$ and $B$ :

$$
y_{A}^{\prime}= \pm \frac{\sqrt{5}}{2} y_{A} \text {, with } y_{A} \text { arbitrary. }
$$

Consider the case $k_{1} \neq 0$ and choose

$$
k_{2}=1, k_{1}=\mp \frac{2}{\sqrt{5}}, k_{0}=1 .
$$

By making use of equation (14), the pair $B$ is obtained in terms of $A$ :

$$
B: y_{B}^{\prime \prime}(t)+\left(\sin (t)+\frac{2}{\sqrt{5}}\right) y_{B}^{\prime}(t)+\left(\frac{\sin ^{2}(t)}{4}+\frac{\sin (t)}{\sqrt{5}}+\frac{\cos (t)}{2}+\frac{3}{4}\right) y_{B}(t)=x_{B}(t) .
$$

Considering the coefficients $b_{2}, b_{1}, b_{0}$ of equation (49) and the system $A$, that is, $a_{2}, a_{1}, a_{0}$ from equation (43), we realize fourth-order LTVS $C$ as 


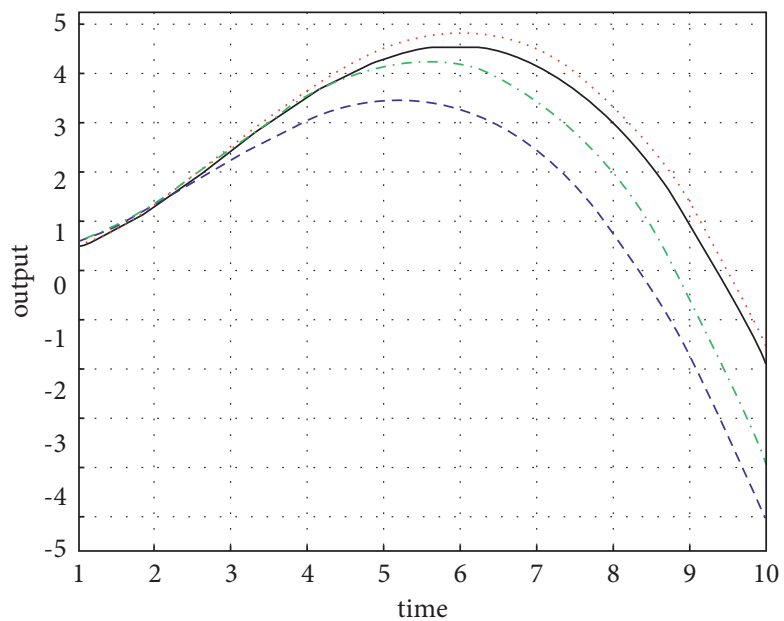

Output Response
- $\mathrm{AB}=\mathrm{BA}=\mathrm{C}$
…. $\mathrm{ABI}$
- - BAI
...- CI

Figure 2: Simulation results of example 1.

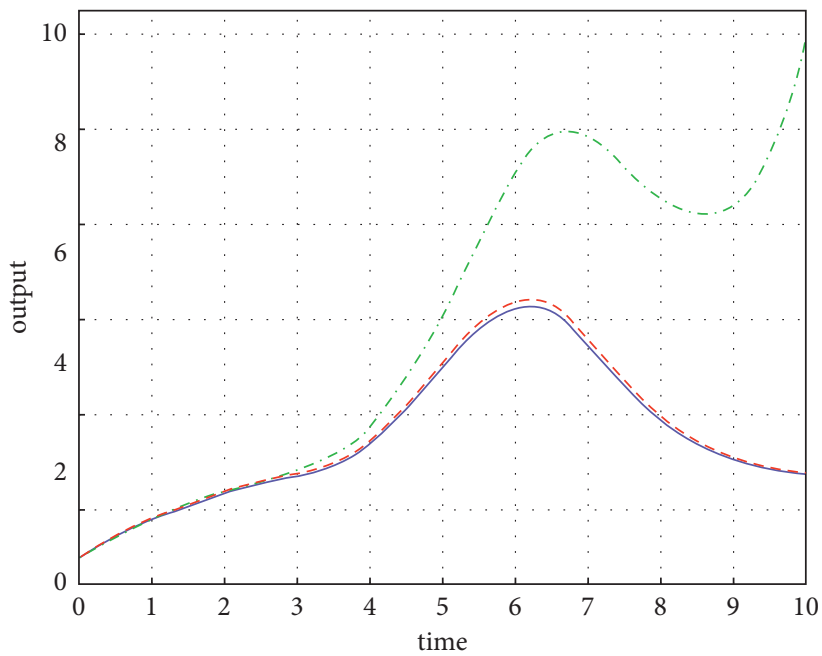

Output Respone

-.- C

- BA

$--\mathrm{AB}$

Figure 3: Simulation results of example 2.

$C: y^{(4)}(t)+\left(2 \sin (t)+\frac{2}{\sqrt{5}}\right) y^{(3)}(t)$

$$
\begin{aligned}
& +\left(\frac{3 \sin ^{2}(t)}{2}+\frac{3 \sin (t)}{\sqrt{5}}+3 \cos (t)+\frac{1}{2}\right) y^{\prime \prime}(t) \\
& +\left(\frac{\sin ^{3}(t)}{2}+\frac{3 \sin ^{2}(t)}{2 \sqrt{5}}+3 \cos (t) \sin (t)+\frac{3 \cos (t)}{\sqrt{5}}-\frac{3 \sin (t)}{2}-\frac{1}{2 \sqrt{5}}\right) y^{\prime}(t) \\
& +\left(\frac{\sin ^{4}(t)}{16}+\frac{\sin ^{3}(t)}{4 \sqrt{5}}+\frac{3}{4} \cos (t) \sin ^{2}(t)+\frac{3 \cos ^{2}(t)}{4}-\frac{7 \sin ^{2}(t)}{8}+\frac{3 \cos (t) \sin (t)}{2 \sqrt{5}}-\frac{\cos (t)}{4}-\frac{\sqrt{5} \sin (t)}{4}-\frac{3}{16}\right)
\end{aligned}
$$

$y(t)=x(t)$. 
Regarding the decomposition with nonzero initial condition, $y\left(t_{0}\right) \neq 0$, equations (16)-(19) yield

$$
\begin{gathered}
y_{B}\left(t_{0}\right)=y_{A}\left(t_{0}\right)=y\left(t_{0}\right), \\
y_{B}^{\prime}\left(t_{0}\right)=y_{A}^{\prime}\left(t_{0}\right)=y^{\prime}\left(t_{0}\right)= \pm \frac{\sqrt{5}}{2} y_{A}\left(t_{0}\right), \\
y_{B}^{\prime \prime}\left(t_{0}\right)=y^{\prime \prime}\left(t_{0}\right)=\frac{3}{4} y\left(t_{0}\right) \text { for } t_{0}=0, \\
y^{(3)}\left(t_{0}\right)=-\frac{1}{4} y^{\prime}\left(t_{0}\right) \text { for } t_{0}=0 .
\end{gathered}
$$

Simulations are carried out with a sinusoid of frequency $3 \mathrm{rad} / \mathrm{sec}$, amplitude 3 , and bias -0.5 . The rationale behind using Runge-Kutta as a solver was based on its numerical stability as well as accuracy. The Runge-Kutta schemes satisfy the possibility condition for any time-step interval "change in time" (see [9] and [10]). Simulink outcomes are illustrated in Figure 3. The initial time and initial conditions chosen are $t_{0}=0$ and $y(0)=y_{A}(0)=y_{B}(0)=-1$, respectively. Computing equations (52)-(54) leads to $y_{B}^{\prime}(0)=y_{A}^{\prime}(0)=y^{\prime}(0)= \pm \sqrt{5} / 2, \quad y^{\prime \prime}(0)=-3 / 4 \quad$ and $y^{(3)}=-5 / 8 \sqrt{5}$. The responses of system $C$ and its decompositions as $A B$ and $B A$ give the same output as shown in Figure $3(C)$. A noise signal in the form of saw-tooth wave (signal generator) with amplitude 0.05 and frequency $0.01 \mathrm{rad} / \mathrm{sec}$ is injected between the subsystems $A$ and $B$. The simulation results are shown in Figure $3(A B$ and $B A)$.

\section{Conclusion}

The commutativity and decomposition of fourth-order Euler LTVS are considered as a commutative pair of two secondorder Euler-type systems. The results are analyzed explicitly and depicted by simulations. Our results highlight the sensitivity of fourth-order Euler-type LTVSs as a result of changes in ICs and present the effect of disturbance due to external noise. Based on these findings, we investigated and discovered that the fourth-order Euler LTVS $C$ possess its commutative pairs $A$ and $B$ which are obtained as a result of the decomposition process in Theorems 1 and 2 as well as in example 1 . The commutative pairs $A$ and $B$ are commutative under certain conditions and can be used for the realization of fourth-order LTVS as seen in example 2. The fourth-order Euler LTVS $C$ in equation (35) and the systems $A B$ and $B A$ of example 1 are sensible toward changes in ICs. Moreover, the system in equation (50) and the systems $A B$ and $B A$ of example 2 show great level of commutativity imbalance toward noise disturbance. Example 1 investigated the decomposition of fourth-order Euler-type LTVS, and our findings verify that fourth-order Euler-type LTVSs always have general decomposed commutative pairs which are also Euler-type systems. The Euler systems possess both constant forward feedback conjugates and non-constant feedback conjugates as commutative pairs. Also, they have less effect toward disturbance due to noise and are sensible toward change in ICs (see [4]). Example 2 presented second-order LTVS as a subsystem that is used for the realization of fourth-order LTVS. The system has a great effect toward disturbance due to noise. These special properties of Euler systems make them a case of interest and differentiate them from other systems.

At last, one can easily observe that the theoretical results are in good agreement with the simulation results. Generalizing and applying these theorems in nonlinear systems, fractional systems, and partial differential systems is an open problem for future investigations. Hence, they remind system designers which connection order should be used in the cascade structure of system synthesis.

\section{Data Availability}

No data were used to support this study.

\section{Conflicts of Interest}

The authors declare that there are no conflicts of interest regarding the publication of this paper.

\section{References}

[1] J. E. Marshall, "Commutativity of time-varying systems," Electronics Letters, vol. 13, no. 18, pp. 539-540, 1977.

[2] M. Köksal, "Commutativity of second order time varying systems," International Journal of Control, vol. 36, no. 3, pp. 541-544, 1982.

[3] M. Koksal, "General conditions for the commutativity of time-varying systems' of second-order time-varying systems," in Proceedings of the International Conference on Telecommunication and Control, pp. 223-225, Halkidiki, Greece, August 1984.

[4] S. Ibrahim and M. E. Koksal, "Commutativity of sixth-order time-varying linear systems," Circuits, Systems, and Signal Processing, vol. 40, no. 10, pp. 4799-4832, 2021.

[5] M. E. Koksal, "Decomposition of a second-order linear timevarying differential system as the series connection of two first order commutative pairs," Open Mathematics, vol. 14, no. 1, pp. 693-704, 2016.

[6] S. Ibrahim and M. E. Koksal, "Realization of a fourth-order linear time-varying differential system with nonzero initial conditions by cascaded two second-order commutative pairs," Circuits, Systems, and Signal Processing, vol. 40, no. 6, pp. 3107-3123, 2021.

[7] M. E. Koksal, "Transitivity of commutativity for second-order linear time-varying analog systems," Circuits, Systems, and Signal Processing, vol. 38, no. 3, pp. 1385-1395, 2019.

[8] A. Silva-Juárez, E. Tlelo-Cuautle, de la Fraga, L. Gerardo, and R. Li, "FPAA-based implementation of fractional-order chaotic oscillators using first-order active filter blocks," Journal of Advanced Research, vol. 25, pp. 77-85, 2020.

[9] K. Sakai, Toward Absolutely Stable Numerical Scheme, LAP LAMBERT Academic Publishing, Moldova, Chisinau, 2015.

[10] M. A. Valencia-Ponce, E. Tlelo-Cuautle, L. G. de la Fraga, and L. G. de la Fraga, "Estimating the highest time-step in numerical methods to enhance the optimization of chaotic oscillators," Mathematics, vol. 9, no. 16, p. 1938, 2021. 